\title{
Open-charm tetraquark candidate: Note on $X_{0}(2900)$
}

\author{
Jian-Rong Zhang® \\ Department of Physics, College of Liberal Arts and Sciences, National University of Defense Technology, \\ Changsha 410073, Hunan, People's Republic of China
}

(Received 31 August 2020; accepted 21 February 2021; published 18 March 2021)

\begin{abstract}
Motivated by the LHCb's very recent observation of exotic $X_{0}(2900)$ in the $B^{+} \rightarrow D^{+} D^{-} K^{+}$process, for which could be a good open-charm $u d \bar{c} \bar{s}$ tetraquark candidate, we endeavor to investigate its possibility by means of QCD sum rules. In technique, four configurations of interpolating currents with $J^{P}=0^{+}$are studied for the $u d \bar{c} \bar{s}$ tetraquark state. In the end, mass values are calculated to be $2.76_{-0.23}^{+0.16} \mathrm{GeV}$ for the axial vector diquark-axial vector antidiquark configuration and $2.75_{-0.24}^{+0.15} \mathrm{GeV}$ for the scalar diquark-scalar antidiquark configuration, both of which are consistent with the experimental data $2.866 \pm 0.007 \pm$ $0.002 \mathrm{GeV}$ of $X_{0}(2900)$ in view of the uncertainty. These results support that $X_{0}(2900)$ could be a $0^{+}$ tetraquark state with open-charm flavor.
\end{abstract}

DOI: 10.1103/PhysRevD.103.054019

\section{INTRODUCTION}

In the past decades, the so-called $X, Y$, and $Z$ new hadrons have attracted wide attentions and some of them were assigned to be possible exotic states (for recent reviews, e.g., see [1-3] and references therein). For example, some prediction was made on a $0^{+}$bound state with a pole mass of $2848 \mathrm{MeV}$ via coupled-channel unitarity [4]. Very recently, the LHCb Collaboration reported the first amplitude analysis of the $B^{+} \rightarrow D^{+} D^{-} K^{+}$decay and included two new exotic structures in the $D^{-} K^{+}$channel with an overwhelming significance [5]. Particularly for the spin-0 resonance $X_{0}(2900)$, its mass and width were measured to be $2.866 \pm$ $0.007 \pm 0.002 \mathrm{GeV}$ and $57 \pm 12 \pm 4 \mathrm{MeV}$, respectively. Taking notice of its decay final states being $D^{-} K^{+}$, $X_{0}(2900)$ was proposed to be a nice open-charm tetraquark candidate [6].

Activated by the LHCb's new experimental result on $X_{0}(2900)$, we attempt to study its possibility to be an opencharm $u d \bar{c} \bar{s}$ tetraquark state. To research into a genuine hadron, one has to face the very complicated nonperturbative QCD problem. As one trustable approach for evaluating nonperturbative effects, the QCD sum rule [7] is firmly established on QCD basic theory and has been successfully applied to numerous hadronic systems [8-12]. For instance, the charm-strange $D_{s 0}^{*}(2317)$ was explored in a tetraquark picture with QCD sum rules [13-19]. In this work, to uncover the internal structure of $X_{0}(2900)$,

Published by the American Physical Society under the terms of the Creative Commons Attribution 4.0 International license. Further distribution of this work must maintain attribution to the author(s) and the published article's title, journal citation, and DOI. Funded by SCOAP ${ }^{3}$. we devote to investigating that whether it could be an opencharm $u d \bar{c} \bar{s}$ tetraquark state by QCD sum rules.

The rest of the paper is organized as follows. In Sec. II, $X_{0}(2900)$ is studied as a tetraquark state via QCD sum rules, followed by numerical analysis and discussions in Sec. III. The last part gives a concise summary.

\section{QCD SUM RULE STUDY OF $X_{0}(2900)$ AS A $0^{+} u d \bar{c} \bar{s}$ TETRAQUARK STATE}

Complying with the usual treatment of QCD sum rules, a tetraquark state can be represented by an interpolating current with the diquark-antidiquark configuration (e.g., see the review [12] and references therein). Particularly for the present $0^{+} u d \bar{c} \bar{s}$ tetraquark state, one can construct its different configuration currents with $0^{+}$composed of an $u d$-diquark and a $\bar{c} \bar{s}$-antidiquark, taking into account that $u_{a}^{T} C \gamma_{5} d_{b}$ as a $0^{+}$scalar diquark, $u_{a}^{T} C d_{b}$ as a $0^{-}$pseudoscalar diquark, $u_{a}^{T} C \gamma_{\mu} d_{b}$ as a $1^{+}$axial vector diquark, $u_{a}^{T} C \gamma_{5} \gamma_{\mu} d_{b}$ as a $1^{-}$vector diquark, and likewise for the $\bar{c} \bar{s}$ antidiquark. In this manner, following forms of currents are presented for the $0^{+} u d \bar{c} \bar{s}$ tetraquark state, with

$$
j_{(1)}=\epsilon_{a b g} \epsilon_{a^{\prime} b^{\prime} g}\left(u_{a}^{T} C \gamma_{5} d_{b}\right)\left(\bar{c}_{a^{\prime}} \gamma_{5} C \bar{s}_{b^{\prime}}^{T}\right)
$$

for the scalar diquark-scalar antidiquark configuration,

$$
j_{(2)}=\epsilon_{a b g} \epsilon_{a^{\prime} b^{\prime} g}\left(u_{a}^{T} C d_{b}\right)\left(\bar{c}_{a^{\prime}} C \bar{s}_{b^{\prime}}^{T}\right)
$$

for the pseudoscalar diquark-pseudoscalar antidiquark configuration,

$$
j_{(3)}=\epsilon_{a b g} \epsilon_{a^{\prime} b^{\prime} g}\left(u_{a}^{T} C \gamma_{\mu} d_{b}\right)\left(\bar{c}_{a^{\prime}} \gamma^{\mu} C \bar{s}_{b^{\prime}}^{T}\right)
$$


for the axial vector diquark-axial vector antidiquark configuration, and

$$
j_{(4)}=\epsilon_{a b g} \epsilon_{a^{\prime} b^{\prime} g}\left(u_{a}^{T} C \gamma_{5} \gamma_{\mu} d_{b}\right)\left(\bar{c}_{a^{\prime}} \gamma^{\mu} \gamma_{5} C \bar{s}_{b^{\prime}}^{T}\right)
$$

for the vector diquark-vector antidiquark configuration. Here $T$ indicates matrix transposition, $C$ is the charge conjugation matrix, and the subscripts $a, b, g, a^{\prime}$, and $b^{\prime}$ are color indices.

Besides, one can construct some other current, such as

$$
j_{(5)}=\epsilon_{a b g} \epsilon_{a^{\prime} b^{\prime} g}\left(u_{a}^{T} C \sigma_{\mu \nu} d_{b}\right)\left(\bar{c}_{a^{\prime}} \sigma^{\mu \nu} C \bar{s}_{b^{\prime}}^{T}\right) .
$$

Meanwhile, one could consider that the corresponding diquark and antidiquark are higher excitation and they are comparatively difficult to be stably formed. Then the concrete calculations for this current are not involved here, for which could be taken into account in some further work.

On the one hand, the two-point correlator

$$
\begin{gathered}
\Pi_{i}\left(q^{2}\right)=i \int d^{4} x \mathrm{e}^{i q \cdot x}\left\langle 0\left|T\left[j_{(i)}(x) j_{(i)}^{\dagger}(0)\right]\right| 0\right\rangle, \\
(i=1,2,3, \text { or } 4)
\end{gathered}
$$

can be phenomenologically expressed as

$$
\Pi_{i}\left(q^{2}\right)=\frac{\lambda_{H}^{2}}{M_{H}^{2}-q^{2}}+\frac{1}{\pi} \int_{s_{0}}^{\infty} \frac{\operatorname{Im}\left[\Pi_{i}^{\text {phen }}(s)\right]}{s-q^{2}} d s,
$$

where $s_{0}$ is the continuum threshold, $M_{H}$ is the hadron's mass, and $\lambda_{H}$ denotes the hadronic coupling constant $\langle 0|j| H\rangle=\lambda_{H}$.

On the other hand, $\Pi_{i}\left(q^{2}\right)$ can be theoretically formalized as

$$
\Pi_{i}\left(q^{2}\right)=\int_{\left(m_{c}+m_{s}\right)^{2}}^{\infty} \frac{\rho_{i}}{s-q^{2}} d s+\Pi_{i}^{\text {cond }}\left(q^{2}\right),
$$

in which $m_{c}$ is the charm mass, $m_{s}$ is the strange mass, and the spectral density $\rho_{i}=\frac{1}{\pi} \operatorname{Im}\left[\Pi_{i}(s)\right]$.

Matching the two equations (7) and (8), assuming quarkhadron duality, and making a Borel transform $\hat{B}$, the sum rule can be written as

$$
\lambda_{H}^{2} e^{-M_{H}^{2} / M^{2}}=\int_{\left(m_{c}+m_{s}\right)^{2}}^{s_{0}} \rho_{i} e^{-s / M^{2}} d s+\hat{B} \Pi_{i}^{\text {cond }},
$$

with the Borel parameter $M^{2}$.

Taking the derivative of the sum rule (9) with $-\frac{1}{M^{2}}$ and then dividing the result by (9) itself, one can get the hadronic mass

$$
M_{H}=\sqrt{\left[\int_{\left(m_{c}+m_{s}\right)^{2}}^{s_{0}} \rho_{i} s e^{-s / M^{2}} d s+\frac{d\left(\hat{B} \Pi_{i}^{\text {cond }}\right)}{d\left(-\frac{1}{M^{2}}\right)}\right] /\left[\int_{\left(m_{c}+m_{s}\right)^{2}}^{s_{0}} \rho_{i} e^{-s / M^{2}} d s+\hat{B} \Pi_{i}^{\text {cond }}\right]},
$$

for which the spectral density $\rho_{i}$ and the term $\hat{B} \Pi_{i}^{\text {cond }}$ can be derived making use of the similar techniques as Refs. [12-14,20], and for concision their expressions are wholly enclosed in the Appendix A.

One could note that there is not the $\langle\bar{q} q\rangle$ or $\langle g \bar{q} \sigma \cdot G q\rangle$ condensate in this work, which is mainly resulted from two aspects of reasons. On the one hand, both light $u$ and $d$ quark masses are so small comparing with the heavy charm mass that light quark masses have been safely neglected, and thus there does not appear the $\langle\bar{q} q\rangle$ or $\langle g \bar{q} \sigma \cdot G q\rangle$ term proportional to $m_{u}$ or $m_{d}$. On the other hand, the $\langle\bar{q} q\rangle$ or $\langle g \bar{q} \sigma \cdot G q\rangle$ term without $m_{u}$ or $m_{d}$ vanishes owing to that its corresponding matrix trace happens to be zero. In this way, the spectral density $\rho$ does not contain the $\langle\bar{q} q\rangle$ or $\langle g \bar{q} \sigma \cdot G q\rangle$ condensate.

\section{NUMERICAL ANALYSIS AND DISCUSSIONS}

To extract the mass $M_{H}$, one can carry out the numerical analysis of sum rule (10), with the aid of input parameters $\langle\bar{q} q\rangle=-(0.24 \pm 0.01)^{3} \mathrm{GeV}^{3}, \quad\langle g \bar{q} \sigma \cdot G q\rangle=m_{0}^{2}\langle\bar{q} q\rangle$, $\langle\bar{s} s\rangle=m_{0}^{2}\langle\bar{q} q\rangle, \quad\langle g \bar{s} \sigma \cdot G s\rangle=m_{0}^{2}\langle\bar{s} s\rangle, \quad m_{0}^{2}=0.8 \pm 0.1 \mathrm{GeV}^{2}$, $\left\langle g^{2} G^{2}\right\rangle=0.88 \pm 0.25 \mathrm{GeV}^{4}$, and $\left\langle g^{3} G^{3}\right\rangle=0.58 \pm 0.18 \mathrm{GeV}^{6}$ [7,9]. Besides, quark masses are taken as $m_{c}=1.27 \pm$ $0.02 \mathrm{GeV}$ and $m_{s}=93_{-5}^{+11} \mathrm{MeV}$ [21], respectively. Keeping to the procedure of sum rule analysis, both the OPE convergence and pole dominance should be inspected to find suitable work windows for the threshold $\sqrt{s_{0}}$ and the Borel parameter $M^{2}$.

Taking the axial vector diquark-axial vector antidiquark case as an example, its various relative OPE contributions are compared in FIG. 1, which displays that there are three main condensate contributions, i.e., the two-quark condensate $\langle\bar{s} s\rangle$, the mixed condensate $\langle\bar{s} g \sigma \cdot G s\rangle$, and the four-quark condensate $\langle\bar{q} q\rangle^{2}$. Comparatively, one could note that the $\langle\bar{q} q\rangle^{2}$ contribution is bigger than the lower dimension condensate like $\langle\bar{s} s\rangle$ or $\langle\bar{s} g \sigma \cdot G s\rangle$. Frankly speaking, this is a common problem existing in some multiquark QCD sum rule studies but not newly arisen, for 


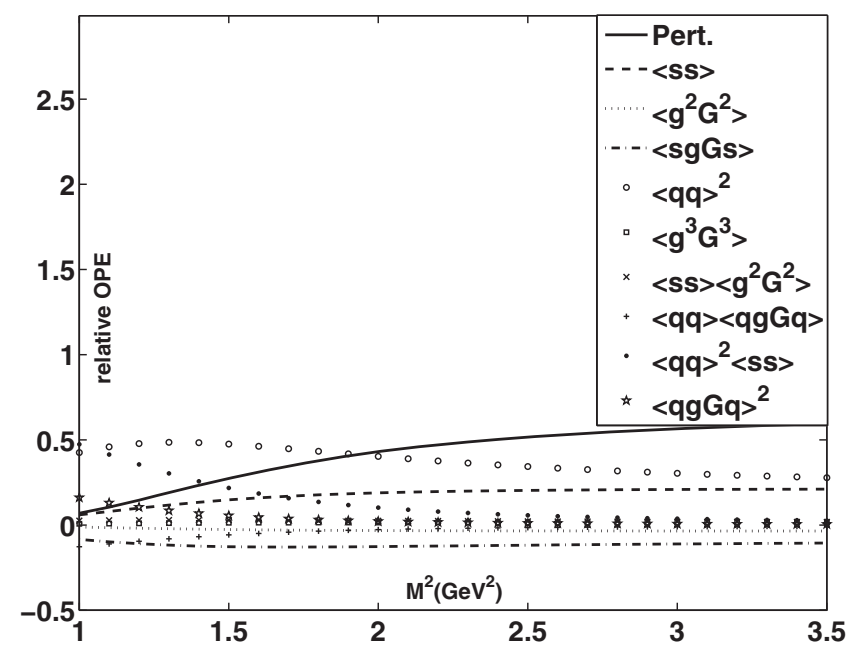

FIG. 1. The relative contributions of various condensates as a function of $M^{2}$ in sum rule (10) for $\sqrt{s_{0}}=3.4 \mathrm{GeV}$ for the axial vector diquark-axial vector antidiquark case.

which has already been discussed in some other works [22-25]. Namely, some individual high dimension condensate (e.g., the $\langle\bar{q} q\rangle^{2}$ here) plays an important role on the OPE side, which causes that it is not easy to satisfy the traditional condition for conventional hadrons that low dimension condensate should be bigger than high dimension one in the OPE. Acceptably, these main condensate contributions can fortunately counteract each other to some extent. One might note that the $\langle\bar{q} q\rangle^{2}\langle\bar{s} s\rangle$ condensate is not small at $M^{2}=1 \mathrm{GeV}^{2}$, however, it descends rapidly with the increase of $M^{2}$ and becomes very small while taking $M^{2} \geq 2.0 \mathrm{GeV}^{2}$. All these factors bring that the lowest

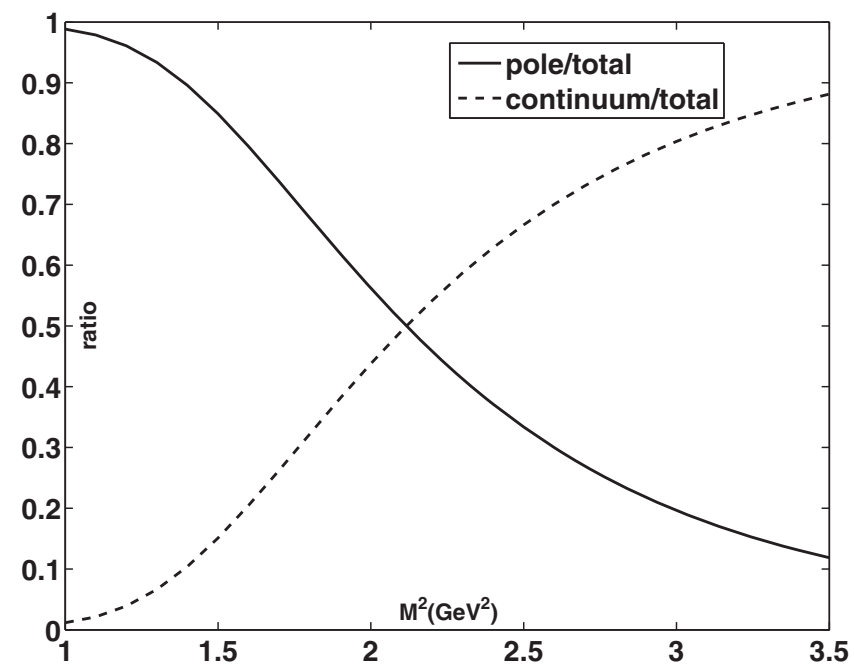

FIG. 2. The phenomenological contribution as a function of $M^{2}$ in sum rule (10) for $\sqrt{s_{0}}=3.4 \mathrm{GeV}$ for the axial vector diquarkaxial vector antidiquark case. The solid line is the relative pole contribution and the dashed line is the relative continuum contribution. dimension perturbative part can play an important role on the total OPE when $M^{2} \geq 2.0 \mathrm{GeV}^{2}$ and the corresponding OPE convergence is still under control in the work windows.

In phenomenology, Fig. 2 shows the comparison between pole contribution and continuum contribution of sum rule (9) for $\sqrt{s_{0}}=3.4 \mathrm{GeV}$, which manifests that the relative pole contribution is close to $50 \%$ at $M^{2}=$ $2.2 \mathrm{GeV}^{2}$ and decreases with $M^{2}$. Thereby, the upper bound of $M^{2}$ is chosen as $2.2 \mathrm{GeV}^{2}$ for $\sqrt{s_{0}}=3.4 \mathrm{GeV}$. Similarly, the upper values of $M^{2}$ can also be achieved for $\sqrt{s_{0}}=3.3 \mathrm{GeV}$ and $\sqrt{s_{0}}=3.5 \mathrm{GeV}$. Accordingly, work windows for the axial vector diquark-axial vector antidiquark case are fixed as $M^{2}=2.0 \sim 2.1 \mathrm{GeV}^{2}$ for $\sqrt{s_{0}}=$ $3.3 \mathrm{GeV}, M^{2}=2.0 \sim 2.2 \mathrm{GeV}^{2}$ for $\sqrt{s_{0}}=3.4 \mathrm{GeV}$, and $M^{2}=2.0 \sim 2.3 \mathrm{GeV}^{2}$ for $\sqrt{s_{0}}=3.5 \mathrm{GeV}$, respectively. In Fig. 3, the mass $M_{H}$ as a function of $M^{2}$ from sum rule (10) is shown for the axial vector diquark-axial vector antidiquark case. Within the chosen work windows, it may seem not very flat for the Borel curves. As one knows, in the choice of work windows, the flatness of Borel curves is an important factor under consideration. Meanwhile, it should not be the only judgement. In fact, the Borel curves can look much flatter if one naively chooses Borel windows with some larger $M^{2}$ here. However, the hypothesis of pole dominance in the phenomenological side of QCD sum rules would be severely broken if overly paying attention to the flatness of Borel curves. In practice, the procedure of finding work windows has actually been developed from the traditional way of mainly observing that wether there is some flat Borel plateau to the present-day way of choosing

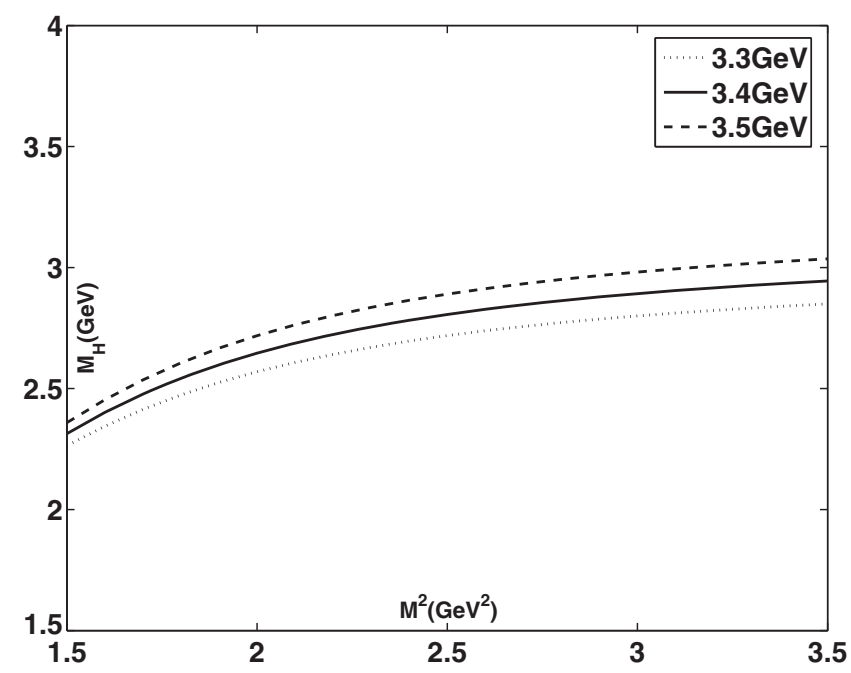

FIG. 3. The mass of $0^{+} u d \bar{c} \bar{s}$ tetraquark state with the axial vector diquark-axial vector antidiquark configuration as a function of $M^{2}$ from sum rule (10). The ranges of $M^{2}$ are taken as $2.0 \sim 2.1 \mathrm{GeV}^{2}$ for $\sqrt{s_{0}}=3.3 \mathrm{GeV}, 2.0 \sim 2.2 \mathrm{GeV}^{2}$ for $\sqrt{s_{0}}=3.4 \mathrm{GeV}$, and $2.0 \sim 2.3 \mathrm{GeV}^{2}$ for $\sqrt{s_{0}}=3.5 \mathrm{GeV}$, respectively. 


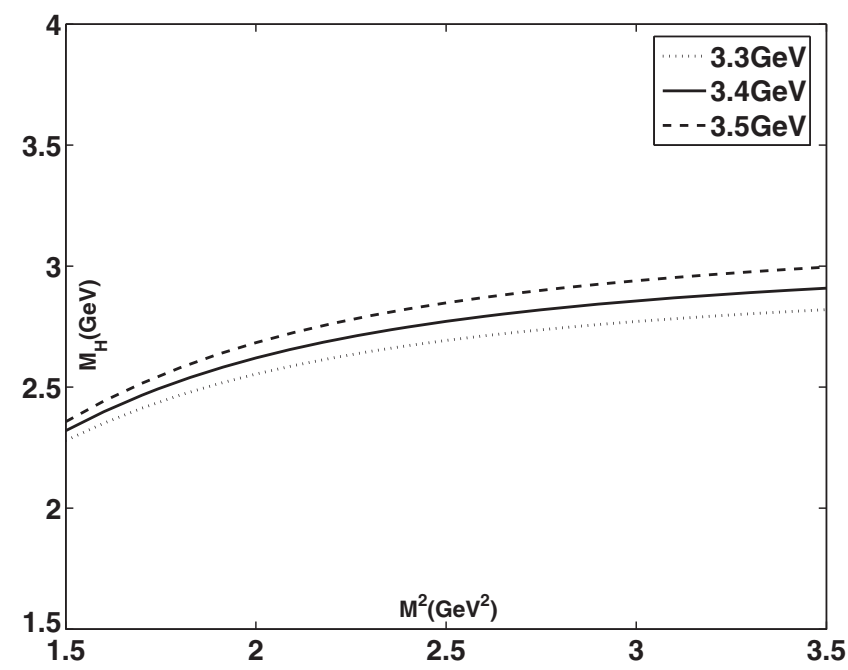

FIG. 4. The mass of $0^{+} u d \bar{c} \bar{s}$ tetraquark state with the scalar diquark-scalar antidiquark configuration as a function of $M^{2}$ from sum rule (10). The ranges of $M^{2}$ are taken as $2.0 \sim 2.2 \mathrm{GeV}^{2}$ for $\sqrt{s_{0}}=3.3 \mathrm{GeV}, 2.0 \sim 2.3 \mathrm{GeV}^{2}$ for $\sqrt{s_{0}}=3.4 \mathrm{GeV}$, and $2.0 \sim 2.4 \mathrm{GeV}^{2}$ for $\sqrt{s_{0}}=3.5 \mathrm{GeV}$, respectively.

suitable work windows fulfilling both the OPE convergence and pole dominance, to ensure that two sides of QCD sum rules have a good overlap and information on the hadronic resonance can be reliably extracted. Moreover, the variation of mass with Borel parameter $M^{2}$ in the Borel curve can be numerically embodied by the uncertainty of final result. After considering the uncertainty from the variation of QCD parameters, one gains the final mass $2.76_{-0.23}^{+0.16} \mathrm{GeV}$ for the axial vector diquark-axial vector antidiquark case.

For the scalar diquark-scalar antidiquark case, through the similar procedure, its Borel windows are determined to be $2.0 \sim 2.2 \mathrm{GeV}^{2}$ for $\sqrt{s_{0}}=3.3 \mathrm{GeV}, 2.0 \sim 2.3 \mathrm{GeV}^{2}$ for $\sqrt{s_{0}}=3.4 \mathrm{GeV}$, and $2.0 \sim 2.4 \mathrm{GeV}^{2}$ for $\sqrt{s_{0}}=3.5 \mathrm{GeV}$, respectively. Furthermore, its mass $M_{H}$ dependence on $M^{2}$ from sum rule (10) is shown in Fig. 4. Including the uncertainty due to QCD parameters, the mass for the scalar diquark-scalar antidiquark configuration is calculated to be $2.75_{-0.24}^{+0.15} \mathrm{GeV}$ at last.

After similar analysis, it is noted that the OPE convergence for the pseudoscalar diquark-pseudoscalar antidiquark and the vector diquark-vector antidiquark cases is so unsatisfactory that one cannot find any appropriate work windows for them and it is not advisable to continue extracting their mass results. Anyway, the final results for the axial vector diquark-axial vector antidiquark and the scalar diquark-scalar antidiquark cases both agree with the experimental data of $X_{0}(2900)$ viewing the uncertainty of final results, which supports that $X_{0}(2900)$ could be a $0^{+}$ $u d \bar{c} \bar{s}$ tetraquark state with the axial vector diquark-axial vector antidiquark or the scalar diquark-scalar antidiquark configuration.

\section{SUMMARY}

Stimulated by the new observation of exotic $X_{0}(2900)$, we explore the possibility of $X_{0}(2900)$ as an open-charm $u d \bar{c} \bar{s}$ tetraquark state with $J^{P}=0^{+}$in the framework of QCD sum rules. Finally, the mass values are computed to be $2.76_{-0.23}^{+0.16} \mathrm{GeV}$ for the axial vector diquark-axial vector antidiquark configuration, and $2.75_{-0.24}^{+0.15} \mathrm{GeV}$ for the scalar diquark-scalar antidiquark configuration, respectively. Considering the uncertainty of these results, they are both in agreement with the experimental data of $X_{0}(2900)$. It supports that $X_{0}(2900)$ could be interpreted as a $0^{+} u d \bar{c} \bar{s}$ tetraquark state, whose configuration could be either the axial vector diquark-axial vector antidiquark or the scalar diquark-scalar antidiquark. In future, it is expected that further experimental observations and theoretical efforts could disclose more information on the nature of $X_{0}(2900)$.

\section{ACKNOWLEDGMENTS}

This work was supported by the National Natural Science Foundation of China under Contracts No. 11475258 and 11675263 , and by the project for excellent youth talents in NUDT.

\section{APPENDIX: THE SPECTRAL DENSITY $\rho_{I}$ AND THE TERM $\hat{B} \Pi_{I}^{\text {COND }}$}

The spectral density $\rho_{i}=\rho_{i}^{\text {pert }}+\rho_{i}^{\langle\bar{s} s\rangle}+\rho_{i}^{\left\langle g^{2} G^{2}\right\rangle}+\rho_{i}^{\langle g \bar{s} \sigma \cdot G s\rangle}+$ $\rho_{i}^{\langle\bar{q} q\rangle^{2}}+\rho_{i}^{\left\langle g^{3} G^{3}\right\rangle}+\rho_{i}^{\langle\bar{s} s\rangle\left\langle g^{2} G^{2}\right\rangle}$ and the term $\hat{B} \Pi_{i}^{\text {cond }}$ are collected below, with

$$
\begin{gathered}
\rho_{1}^{\text {pert }}=\frac{1}{3 \cdot 2^{10} \pi^{6}} \int_{\Lambda}^{1} d \alpha\left(\frac{1-\alpha}{\alpha}\right)^{3}\left(\alpha s-m_{c}^{2}+4 m_{s} m_{c}\right) \\
\quad \times\left(\alpha s-m_{c}^{2}\right)^{3}, \\
\begin{aligned}
\rho_{1}^{\langle\bar{s} s\rangle}= & -\frac{\langle\bar{s} s\rangle}{2^{6} \pi^{4}} \int_{\Lambda}^{1} d \alpha \frac{1-\alpha}{\alpha^{2}}\left[(1-\alpha) m_{c}-\alpha m_{s}\right]\left(\alpha s-m_{c}^{2}\right)^{2},
\end{aligned} \\
\rho_{1}^{\left\langle g^{2} G^{2}\right\rangle}=-\frac{m_{c}\left\langle g^{2} G^{2}\right\rangle}{3^{2} \cdot 2^{10} \pi^{6}} \int_{\Lambda}^{1} d \alpha\left(\frac{1-\alpha}{\alpha}\right)^{3} \\
\quad \times\left[\left(m_{c}-3 m_{s}\right)\left(\alpha s-m_{c}^{2}\right)+m_{s} m_{c}^{2}\right], \\
\rho_{1}^{\langle g \bar{s} \sigma \cdot G s\rangle}=\frac{\langle g \bar{s} \sigma \cdot G s\rangle}{3 \cdot 2^{6} \pi^{4}} \int_{\Lambda}^{1} \frac{d \alpha}{\alpha}\left[3(1-\alpha) m_{c}-\alpha m_{s}\right]\left(\alpha s-m_{c}^{2}\right), \\
\rho_{1}^{\langle\bar{q} q\rangle^{2}}=\frac{\langle\bar{q} q\rangle^{2}}{3 \cdot 2^{2} \pi^{2}} \int_{\Lambda}^{1} d \alpha\left[\left(\alpha s-m_{c}^{2}\right)+m_{s} m_{c}\right], \\
\rho_{1}^{\left\langle g^{3} G^{3}\right\rangle}=-\frac{\left\langle g^{3} G^{3}\right\rangle}{3^{2} \cdot 2^{12} \pi^{6}} \int_{\Lambda}^{1} d \alpha\left(\frac{1-\alpha}{\alpha}\right)^{3}\left(\alpha s-3 m_{c}^{2}+6 m_{s} m_{c}\right),
\end{gathered}
$$


$\rho_{1}^{\langle\bar{s} s\rangle\left\langle g^{2} G^{2}\right\rangle}=-\frac{m_{c}\langle\bar{s} s\rangle\left\langle g^{2} G^{2}\right\rangle}{3^{2} \cdot 2^{8} \pi^{4}} \int_{\Lambda}^{1} d \alpha\left[1+3\left(\frac{1-\alpha}{\alpha}\right)^{2}\right]$,

$$
\begin{aligned}
\hat{B} \Pi_{1}^{\text {cond }}= & -\frac{m_{s} m_{Q}\langle\bar{q} q\rangle\langle g \bar{q} \sigma \cdot G q\rangle}{3 \cdot 2^{3} \pi^{2}} e^{-m_{Q}^{2} / M^{2}} \\
& -\frac{\langle\bar{q} q\rangle^{2}\langle\bar{s} s\rangle}{2 \cdot 3^{2}}\left(2 m_{Q}-m_{s}-\frac{m_{s} m_{Q}^{2}}{M^{2}}\right) e^{-m_{Q}^{2} / M^{2}} \\
& +\frac{\langle g \bar{q} \sigma \cdot G q\rangle^{2}}{3 \cdot 2^{6} \pi^{2}}\left[1+\frac{m_{Q}^{2}}{M^{2}}+\frac{m_{s} m_{Q}^{3}}{\left(M^{2}\right)^{2}}\right] e^{-m_{Q}^{2} / M^{2}}
\end{aligned}
$$

for the scalar diquark-scalar antidiquark case,

$$
\begin{aligned}
\rho_{2}^{\text {pert }}= & \frac{1}{3 \cdot 2^{10} \pi^{6}} \int_{\Lambda}^{1} d \alpha\left(\frac{1-\alpha}{\alpha}\right)^{3}\left(\alpha s-m_{c}^{2}-4 m_{s} m_{c}\right) \\
& \times\left(\alpha s-m_{c}^{2}\right)^{3}, \\
\rho_{2}^{\langle\bar{s} s\rangle}= & \frac{\langle\bar{s} s\rangle}{2^{6} \pi^{4}} \int_{\Lambda}^{1} d \alpha \frac{1-\alpha}{\alpha^{2}}\left[(1-\alpha) m_{c}+\alpha m_{s}\right]\left(\alpha s-m_{c}^{2}\right)^{2},
\end{aligned}
$$

$$
\begin{aligned}
\rho_{2}^{\left\langle g^{2} G^{2}\right\rangle}= & -\frac{m_{c}\left\langle g^{2} G^{2}\right\rangle}{3^{2} \cdot 2^{10} \pi^{6}} \int_{\Lambda}^{1} d \alpha\left(\frac{1-\alpha}{\alpha}\right)^{3} \\
& \times\left[\left(m_{c}+3 m_{s}\right)\left(\alpha s-m_{c}^{2}\right)-m_{s} m_{c}^{2}\right], \quad(\mathrm{A} 11) \\
\rho_{2}^{\langle g \bar{s} \sigma \cdot G s\rangle}= & -\frac{\langle g \bar{s} \sigma \cdot G s\rangle}{3 \cdot 2^{6} \pi^{4}} \int_{\Lambda}^{1} \frac{d \alpha}{\alpha}\left[3(1-\alpha) m_{c}+\alpha m_{s}\right] \\
& \times\left(\alpha s-m_{c}^{2}\right), \\
\rho_{2}^{\langle\bar{q} q\rangle^{2}}= & \frac{\langle\bar{q} q\rangle^{2}}{3 \cdot 2^{2} \pi^{2}} \int_{\Lambda}^{1} d \alpha\left[-\left(\alpha s-m_{c}^{2}\right)+m_{s} m_{c}\right], \quad(\mathrm{A} 13) \\
\rho_{2}^{\left\langle g^{3} G^{3}\right\rangle}=- & \frac{\left\langle g^{3} G^{3}\right\rangle}{3^{2} \cdot 2^{12} \pi^{6}} \int_{\Lambda}^{1} d \alpha\left(\frac{1-\alpha}{\alpha}\right)^{3}\left(\alpha s-3 m_{c}^{2}-6 m_{s} m_{c}\right),
\end{aligned}
$$

$$
\rho_{2}^{\langle\bar{s} s\rangle\left\langle g^{2} G^{2}\right\rangle}=\frac{m_{c}\langle\bar{s} s\rangle\left\langle g^{2} G^{2}\right\rangle}{3^{2} \cdot 2^{8} \pi^{4}} \int_{\Lambda}^{1} d \alpha\left[1+3\left(\frac{1-\alpha}{\alpha}\right)^{2}\right]
$$

$$
\begin{aligned}
\hat{B} \Pi_{2}^{\text {cond }}= & -\frac{m_{s} m_{Q}\langle\bar{q} q\rangle\langle g \bar{q} \sigma \cdot G q\rangle}{3 \cdot 2^{3} \pi^{2}} e^{-m_{Q}^{2} / M^{2}} \\
& -\frac{\langle\bar{q} q\rangle^{2}\langle\bar{s} s\rangle}{2 \cdot 3^{2}}\left(2 m_{Q}+m_{s}+\frac{m_{s} m_{Q}^{2}}{M^{2}}\right) e^{-m_{Q}^{2} / M^{2}} \\
& +\frac{\langle g \bar{q} \sigma \cdot G q\rangle^{2}}{3 \cdot 2^{6} \pi^{2}}\left[-1-\frac{m_{Q}^{2}}{M^{2}}+\frac{m_{s} m_{Q}^{3}}{\left(M^{2}\right)^{2}}\right] e^{-m_{Q}^{2} / M^{2}},
\end{aligned}
$$

for the pseudoscalar diquark-pseudoscalar antidiquark case,

$$
\begin{aligned}
\rho_{3}^{\text {pert }}= & \frac{1}{3 \cdot 2^{8} \pi^{6}} \int_{\Lambda}^{1} d \alpha\left(\frac{1-\alpha}{\alpha}\right)^{3}\left(\alpha s-m_{c}^{2}+2 m_{s} m_{c}\right) \\
& \times\left(\alpha s-m_{c}^{2}\right)^{3}, \\
\rho_{3}^{\langle\bar{s} s\rangle}= & -\frac{\langle\bar{s} s\rangle}{2^{5} \pi^{4}} \int_{\Lambda}^{1} d \alpha \frac{1-\alpha}{\alpha^{2}}\left[(1-\alpha) m_{c}-2 \alpha m_{s}\right]\left(\alpha s-m_{c}^{2}\right)^{2}, \\
\rho_{3}^{\left\langle g^{2} G^{2}\right\rangle}= & -\frac{m_{c}\left\langle g^{2} G^{2}\right\rangle}{3^{2} \cdot 2^{9} \pi^{6}} \int_{\Lambda}^{1} d \alpha\left(\frac{1-\alpha}{\alpha}\right)^{3} \\
& \times\left[\left(2 m_{c}-3 m_{s}\right)\left(\alpha s-m_{c}^{2}\right)+m_{s} m_{c}^{2}\right], \\
\rho_{3}^{\langle g \bar{s} \sigma \cdot G s\rangle}= & \frac{\langle g \bar{s} \sigma \cdot G s\rangle}{3 \cdot 2^{5} \pi^{4}} \int_{\Lambda}^{1} \frac{d \alpha}{\alpha}\left[3(1-\alpha) m_{c}-2 \alpha m_{s}\right]\left(\alpha s-m_{c}^{2}\right),
\end{aligned}
$$

$$
\begin{gathered}
\rho_{3}^{\langle\bar{q} q\rangle^{2}}=\frac{\langle\bar{q} q\rangle^{2}}{3 \cdot 2 \pi^{2}} \int_{\Lambda}^{1} d \alpha\left[\left(\alpha s-m_{c}^{2}\right)+2 m_{s} m_{c}\right], \\
\rho_{3}^{\left\langle g^{3} G^{3}\right\rangle}=-\frac{\left\langle g^{3} G^{3}\right\rangle}{3^{2} \cdot 2^{10} \pi^{6}} \int_{\Lambda}^{1} d \alpha\left(\frac{1-\alpha}{\alpha}\right)^{3}\left(\alpha s-3 m_{c}^{2}+3 m_{s} m_{c}\right), \\
\rho_{3}^{\langle\bar{s} s\rangle\left\langle g^{2} G^{2}\right\rangle}=-\frac{m_{c}\langle\bar{s} s\rangle\left\langle g^{2} G^{2}\right\rangle}{3^{2} \cdot 2^{7} \pi^{4}} \int_{\Lambda}^{1} d \alpha\left[1+3\left(\frac{1-\alpha}{\alpha}\right)^{2}\right]
\end{gathered}
$$

$$
\begin{aligned}
\hat{B} \Pi_{3}^{\text {cond }}= & -\frac{m_{s} m_{Q}\langle\bar{q} q\rangle\langle g \bar{q} \sigma \cdot G q\rangle}{3 \cdot 2 \pi^{2}} e^{-m_{Q}^{2} / M^{2}} \\
& -\frac{\langle\bar{q} q\rangle^{2}\langle\bar{s} s\rangle}{3^{2}}\left(4 m_{Q}-m_{s}-\frac{m_{s} m_{Q}^{2}}{M^{2}}\right) e^{-m_{Q}^{2} / M^{2}} \\
& +\frac{\langle g \bar{q} \sigma \cdot G q\rangle^{2}}{3 \cdot 2^{5} \pi^{2}}\left[1+\frac{m_{Q}^{2}}{M^{2}}+2 \frac{m_{s} m_{Q}^{3}}{\left(M^{2}\right)^{2}}\right] e^{-m_{Q}^{2} / M^{2}},
\end{aligned}
$$

for the axial vector diquark-axial vector antidiquark case, and

$$
\begin{aligned}
\rho_{4}^{\text {pert }}= & \frac{1}{3 \cdot 2^{8} \pi^{6}} \int_{\Lambda}^{1} d \alpha\left(\frac{1-\alpha}{\alpha}\right)^{3}\left(\alpha s-m_{c}^{2}-2 m_{s} m_{c}\right) \\
& \times\left(\alpha s-m_{c}^{2}\right)^{3}, \\
\rho_{4}^{\langle\bar{s} s\rangle}= & \frac{\langle\bar{s} s\rangle}{2^{5} \pi^{4}} \int_{\Lambda}^{1} d \alpha \frac{1-\alpha}{\alpha^{2}}\left[(1-\alpha) m_{c}+2 \alpha m_{s}\right]\left(\alpha s-m_{c}^{2}\right)^{2},
\end{aligned}
$$




$$
\begin{aligned}
\rho_{4}^{\left\langle g^{2} G^{2}\right\rangle}= & -\frac{m_{c}\left\langle g^{2} G^{2}\right\rangle}{3^{2} \cdot 2^{9} \pi^{6}} \int_{\Lambda}^{1} d \alpha\left(\frac{1-\alpha}{\alpha}\right)^{3} \\
& \times\left[\left(2 m_{c}+3 m_{s}\right)\left(\alpha s-m_{c}^{2}\right)-m_{s} m_{c}^{2}\right], \quad(\mathrm{A} 27) \\
\rho_{4}^{\langle g \bar{s} \cdot G s\rangle}= & -\frac{\langle g \bar{s} \sigma \cdot G s\rangle}{3 \cdot 2^{5} \pi^{4}} \int_{\Lambda}^{1} \frac{d \alpha}{\alpha}\left[3(1-\alpha) m_{c}+2 \alpha m_{s}\right] \\
& \times\left(\alpha s-m_{c}^{2}\right), \\
\rho_{4}^{\langle\bar{q} q\rangle^{2}}= & \frac{\langle\bar{q} q\rangle^{2}}{3 \cdot 2 \pi^{2}} \int_{\Lambda}^{1} d \alpha\left[-\left(\alpha s-m_{c}^{2}\right)+2 m_{s} m_{c}\right], \quad(\mathrm{A} 29) \\
\rho_{4}^{\left\langle g^{3} G^{3}\right\rangle}= & -\frac{\left\langle g^{3} G^{3}\right\rangle}{3^{2} \cdot 2^{10} \pi^{6}} \int_{\Lambda}^{1} d \alpha\left(\frac{1-\alpha}{\alpha}\right)^{3}\left(\alpha s-3 m_{c}^{2}-3 m_{s} m_{c}\right), \\
\rho_{4}^{\langle\bar{s} s\rangle\left\langle g^{2} G^{2}\right\rangle}= & \frac{m_{c}\langle\bar{s} s\rangle\left\langle g^{2} G^{2}\right\rangle}{3^{2} \cdot 2^{7} \pi^{4}} \int_{\Lambda}^{1} d \alpha\left[1+3\left(\frac{1-\alpha}{\alpha}\right)^{2}\right],
\end{aligned}
$$

$$
\begin{aligned}
\hat{B} \Pi_{4}^{\text {cond }}= & -\frac{m_{s} m_{Q}\langle\bar{q} q\rangle\langle g \bar{q} \sigma \cdot G q\rangle}{3 \cdot 2 \pi^{2}} e^{-m_{Q}^{2} / M^{2}} \\
& -\frac{\langle\bar{q} q\rangle^{2}\langle\bar{s} s\rangle}{3^{2}}\left(4 m_{Q}+m_{s}+\frac{m_{s} m_{Q}^{2}}{M^{2}}\right) e^{-m_{Q}^{2} / M^{2}} \\
& +\frac{\langle g \bar{q} \sigma \cdot G q\rangle^{2}}{3 \cdot 2^{5} \pi^{2}}\left[-1-\frac{m_{Q}^{2}}{M^{2}}+2 \frac{m_{s} m_{Q}^{3}}{\left(M^{2}\right)^{2}}\right] e^{-m_{Q}^{2} / M^{2}},
\end{aligned}
$$

for the vector diquark-vector antidiquark case. For brevity, the light quark condensate $\langle\bar{u} u\rangle$ or $\langle\bar{d} d\rangle$ is uniformly represented by $\langle\bar{q} q\rangle$, and the integration limit is defined as $\Lambda=m_{c}^{2} / s$. Some high dimension condensates are included above, e.g., $\langle\bar{q} q\rangle\langle g \bar{q} \sigma \cdot G q\rangle,\langle\bar{q} q\rangle^{2}\langle\bar{s} s\rangle$, and $\langle g \bar{q} \sigma \cdot G q\rangle^{2}$. In the chosen work windows, the relative contributions of these kind condensates are very small, and they merely affect the final results in some sort. Most of the other high dimension condensates are formed into some similar terms with negative powers of $q^{2}$, which will be strongly suppressed after making a Borel transform. Similarly, from the work [19] on studying $D_{s 0}^{*}(2317)$ as a $q s \bar{q} \bar{c}$ tetraquark, one can see that high dimension contributions are rather small.
[1] H. X. Chen, W. Chen, X. Liu, Y. R. Liu, and S. L. Zhu, Rep. Prog. Phys. 80, 076201 (2017).

[2] F. K. Guo, C. Hanhart, U.-G. Meißer, Q. Wang, Q. Zhao, and B. S. Zou, Rev. Mod. Phys. 90, 015004 (2018).

[3] A. Esposito, A. Pilloni, and A. D. Polosa, Phys. Rep. 668, 1 (2017).

[4] R. Molina, T. Branz, and E. Oset, Phys. Rev. D 82, 014010 (2010).

[5] R. Aaij et al. (LHCb Collaboration), Phys. Rev. D 102, 112003 (2020); arXiv:2009.00025.

[6] M. Karliner and J. L. Rosner, Phys. Rev. D 102, 094016 (2020).

[7] M. A. Shifman, A. I. Vainshtein, and V. I. Zakharov, Nucl. Phys. B147, 385 (1979); B147, 448 (1979); V. A. Novikov, M. A. Shifman, A. I. Vainshtein, and V.I. Zakharov, Fortschr. Phys. 32, 585 (1984).

[8] B. L. Ioffe, in The Spin Structure of The Nucleon, edited by B. Frois, V. W. Hughes, and N. de Groot (World Scientific, Singapore, 1997).

[9] S. Narison, Cambridge Monogr. Part. Phys., Nucl. Phys., Cosmol. 17, 1 (2002), arXiv:hep-ph/0205006.

[10] P. Colangelo and A. Khodjamirian, in At the Frontier of Particle Physics: Handbook of QCD, edited by M. Shifman, Boris Ioffe Festschrift Vol. 3 (World Scientific, Singapore, 2001), pp. 1495-1576.

[11] L. J. Reinders, H. R. Rubinstein, and S. Yazaki, Phys. Rep. 127, 1 (1985).
[12] M. Nielsen, F. S. Navarra, and S. H. Lee, Phys. Rep. 497, 41 (2010).

[13] M. E. Bracco, A. Lozea, R. D. Matheus, F. S. Navarra, and M. Nielsen, Phys. Lett. B 624, 217 (2005).

[14] H. Kim and Y. Oh, Phys. Rev. D 72, 074012 (2005).

[15] Z. G. Wang and S. L. Wan, Nucl. Phys. A778, 22 (2006).

[16] R. Albuquerque, S. Narison, A. Rabemananjara, and D. Rabetiarivony, Int. J. Mod. Phys. A 31, 1650093 (2016).

[17] W. Chen, H. X. Chen, X. Liu, T. G. Steele, and S. L. Zhu, Phys. Rev. D 95, 114005 (2017).

[18] S. S. Agaev, K. Azizi, and H. Sundu, Eur. Phys. J. C 78, 141 (2018); K. Azizi and U. Özdem, J. Phys. G 45, 055003 (2018).

[19] J. R. Zhang, Phys. Lett. B 789, 432 (2019).

[20] J. R. Zhang, Phys. Rev. D 87, 076008 (2013); 89, 096006 (2014); Eur. Phys. J. C 79, 1001 (2019); J. R. Zhang, J. L. Zou, and J. Y. Wu, Chin. Phys. C 42, 043101 (2018).

[21] M. Tanabashi et al. (Particle Data Group), Phys. Rev. D 98, 030001 (2018) and 2019 update.

[22] R. D. Matheus, F. S. Navarra, M. Nielsen, and R. Rodrigues da Silva, Phys. Rev. D 76, 056005 (2007).

[23] H. X. Chen, A. Hosaka, and S. L. Zhu, Phys. Lett. B 650, 369 (2007).

[24] Z. G. Wang, Nucl. Phys. A791, 106 (2007).

[25] J. R. Zhang, L. F. Gan, and M. Q. Huang, Phys. Rev. D 85, 116007 (2012); J. R. Zhang and G. F. Chen, Phys. Rev. D 86, 116006 (2012); J. R. Zhang, Phys. Rev. D 87, 116004 (2013). 